\title{
Le leadership comme vecteur d'adaptation : Proposition d'un modèle exploratoire de conduite du changement
}

\author{
Julien Bousquet, Éric Jean et Christophe Leyrie \\ Université du Québec à Chicoutimi
}

\section{Introduction}

Il est maintenant acquis que l'environnement économique, longtemps marqué par une forte demande, est aujourd'hui caractérisé par une conjoncture où l'offre est largement supérieure à la demande solvable existante. Cette évolution a été accompagnée par le développement considérable des technologies, et en particulier des technologies de l'information, contribuant ainsi à créer pour les entreprises un environnement à la fois plus global grâce à l'ouverture des marchés locaux, mais aussi plus restreints au travers de la multiplication des concurrents sur ces mêmes marchés. Ces bouleversements ont amené théoriciens comme praticiens à reconnaître de façon unanime que la majorité des entreprises est maintenant passée d'un contexte stable et prévisible à un contexte instable et incertain. On cite aujourd'hui comme une évidence que la seule constante, c'est le changement.

Dans ce contexte, la capacité d'adaptation constitue pour les entreprises l'une des variables clefs de pérennité. Cette pression à l'adaptation se traduit en retour pour les gestionnaires comme pour les autres acteurs de l'organisation par une remise en question de leur rôle qui doit désormais s'exercer dans l'incertitude et la contingence. Chacun doit en effet, à son niveau et dans le cadre de ses responsabilités, devenir un agent de changement efficace. Dans cet environnement complexe, les acteurs impliqués éprouveront inévitablement des difficultés. Certaines de ces difficultés pourront être d'ordre plus technique, alors que d'autres pourront être d'ordre personnel ou relationnel. Dans tous les cas, ces difficultés s'intègrent au contexte social de l'entreprise. La résolution de ces problèmes « sociaux », c'est-à-dire tels qu'ils se présentent aux acteurs dans leur environnement de travail, devient ainsi essentielle à un leadership efficace.

Cet article a pour but de proposer un modèle exploratoire de conduite du changement devant permettre au gestionnaire de mettre en place les conditions favorables à l'adaptation de son organisation. Notre approche repose sur un vecteur ${ }^{1}$ principal, le leadership. Nous proposons d'envisager le «leadership » dans une perspective à la fois individuelle et collective où celui-ci n'est pas uniquement l'apanage du leader, mais également la résultante de relations complexes et dynamiques entre les agents impliqués dans la mise en œuvre du changement ${ }^{2}$. Ce leadership « émergeant», qui transcende le leader, est approché par le biais de trois variables interactives : la résolution de problème, la coopération et la passion. Il est suggéré que ces variables, par leur influence mutuelle, permettent au leader d'avoir un impact sur le complexe réseau de relations au sein de son organisation. Ainsi, la résolution de problèmes, lorsque supportée par une action hautement collective, favorise la créativité nécessaire à l'adaptation. La passion est quant à elle intégrée au modèle comme variable favorisant l'identité collective par son pouvoir d'intégration et d'alignement d'intérêts souvent opposés. Ce cadre de référence "partagé » contribue alors à la mise en place de conditions propices à la conduite du changement. Chacune des constituantes de cet ensemble est présentée dans cette perspective. 


\section{Résolution de problème}

L'un des moyens permettant de mieux comprendre les stratégies personnelles utilisées par le gestionnaire, lorsque confronté à une situation problématique et complexe, consiste à déterminer le type d'approche privilégié. Selon d'Zurilla et ses collaborateurs, deux approches peuvent généralement être utilisées : l'une constructive et l'autre dysfonctionnelle ${ }^{3}$. Ces deux approches n'ont pas les mêmes retombées en matière de résultats. La première, constructive et efficace, présuppose une orientation positive quant aux situations difficiles, ce qui facilite par la suite la résolution rationnelle des problèmes et produit des résultats favorables. Un leader ayant une orientation positive entrevoit le problème comme un défi et estime posséder les habiletés et les ressources qui lui permettront de le relever. De plus, un leader présentant une approche hautement constructive relativement aux problèmes aura une propension, en cas de résultat négatif, à persister dans le processus ou à reprendre le cycle de résolution de problèmes jusqu'à ce qu'une solution satisfaisante émerge et que des résultats positifs soient observés dans le milieu.

Dans la seconde approche, dite dysfonctionnelle, une orientation négative par rapport aux problèmes poussera le gestionnaire à agir plus promptement sans trop se poser de questions. Cette orientation négative se traduit par une appréhension des problèmes, qui sont alors perçus comme une menace au bien-être (psychologique, social ou économique), et comme un défi insurmontable compte tenu de ses habiletés. Ainsi, quoique ce dernier puisse réellement souhaiter voir le problème se régler, les stratégies ou techniques utilisées sont plus impulsives, donc moins réfléchies. Il peut également choisir d'éviter le problème le plus longtemps possible en espérant qu'il se règle de lui-même. Cette façon de résoudre le problème peut également se caractériser par une procrastination marquée ou le transfert de la responsabilité aux autres. De plus, un gestionnaire présentant une approche hautement dysfonctionnelle relativement aux problèmes aura une propension à abandonner ses efforts en cas de résultat négatif. En matière d'adaptation au changement, une telle approche se montre beaucoup moins efficace que l'approche fonctionnelle adoptée par le leader confronté à des difficultés semblables.

\section{La résolution créative des problèmes}

L'efficacité d'un leader au quotidien dépend donc de son attitude par rapport aux problèmes ainsi que des stratégies qui seront mises en œuvre pour les résoudre. Toutefois, les problèmes d'aujourd'hui ne peuvent pas toujours être résolus à partir de notre expérience passée, et les problèmes de demain nécessiteront des paradigmes dissemblables. Ainsi, le leader sait qu'il devra faire les choses autrement. En effet, les défis seront d'un autre ordre et il se verra confronté à des problèmes nouveaux et mal définis. Cela nécessitera une réflexion créative quant aux problèmes. Dans ce contexte, une stratégie « rationnelle» ne saura suffire à la tâche. Le leader devra démontrer des habiletés à « casser » ses paradigmes afin d'en créer d'autres. Cette résolution «créative » exigera le recours à des stratégies collectives de résolution de problème et une orientation constructive de la part de tous les acteurs. C'est en effet par cette action collective et créative qu'un leadership émergeant pourra apparaître.

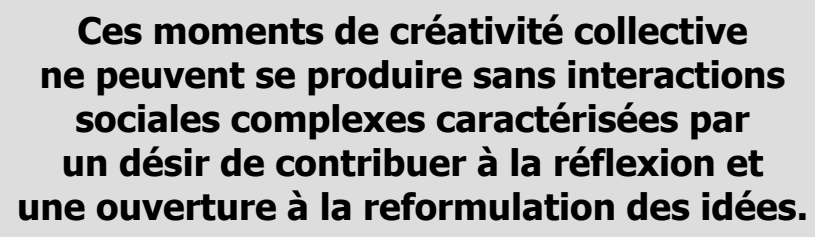

\section{La résolution de problèmes collective}

En effet, même si la résolution créative des problèmes doit d'abord être familière au leader, ce dernier ne saurait faire preuve d'individualisme sans risque. Sans négliger l'importance de la contribution créative du leader en période de changement, il faut reconnaître que la créativité émerge souvent d'un processus collectif. Ces moments de créativité collective ne peuvent se produire sans interactions sociales complexes caractérisées par un désir de contribuer à la réflexion et une ouverture à la reformulation des idées ${ }^{4}$. Cette coopération, constamment renforcée, permet une résolution efficace et créative des problèmes rencontrés par le leader.

\section{La coopération}

La capacité de résoudre collectivement les problèmes posés par l'adaptation aux turbulences de l'environnement confronte donc le gestionnaire au 
défi de la coopération. De façon fondamentale, ce défi renvoie à la nécessité de coordonner les activités des individus et groupes, formés par la division du travail, vers l'atteinte des buts et objectifs organisationnels. Cette coordination se matérialise par des degrés divers de collaboration de la part des individus pouvant aller, si l'on exclut le refus de collaborer, de la participation à la coopération. Un travailleur participatif manifestera des comportements limités à la collaboration prescrite alors qu'un travailleur coopératif fera preuve d'initiative allant au-delà du prescrit, démontrant par là même un niveau supérieur d'investissement dans l'action collective. On dira ici, pour reprendre les termes de Zarafian $^{5}$, que coopérer, c'est « travailler ensemble, développer tout un espace d'intersubjectivité, c'està-dire une compréhension réciproque et des accords solides sur la nature des problèmes à traiter et des savoirs à développer, l'identité des objectifs, le sens donné aux actions et la convergence des mobiles des individus qui agissent ensemble (qui est beaucoup plus que la simple convergence des actes) ».

Quelle que soit l'approche, il appartiendra donc au leader de créer un cadre de référence suffisamment partagé pour que chacun puisse $y$ ancrer les modalités de sa coopération.

\section{La coopération pour le changement}

Dans les environnements plutôt caractérisés par la stabilité, tels que ceux que l'on a connus jusqu'aux années 80 , l'ajustement du système organisationnel à son environnement se fait essentiellement de manière ponctuelle à travers le choix de structures appropriées. L'efficacité de ces structures, généralement centralisées, repose avant tout sur l'adhésion des travailleurs aux règles prescrites, et donc sur une coordination basée sur la participation. En revanche, les contextes de changements vécus par les organisations aujourd'hui imposent à ces dernières un effort d'adaptation constant. Afin d'être efficaces, les organisations, maintenant décentralisées et largement ouvertes sur leurs environnements, doivent pouvoir compter sur des ressources responsabilisées et autonomes évoluant au sein de collectifs de travail. Les nombreux changements impliqués par l'effort de flexibilité et d'adaptation requièrent notamment de la part de ces ressources un investisse- ment supérieur leur permettant de développer une habileté de résolution de problèmes non seulement collective, mais aussi créative. On attendra donc des travailleurs une réelle coopération telle que définie plus haut.

\section{La coopération par l'intégration des intérêts multiples}

La résolution des problèmes inhérents à l'adaptation passe ainsi par la volonté des travailleurs à s'investir dans l'effort de changement et par la capacité des gestionnaires à susciter cette volonté. C'est bien là désormais l'un des défis principaux du leadership, spécifiquement dans les situations particulières connues par la plupart des organisations aujourd'hui. Prétendre que la simple modification des structures pourrait naturellement suffire au changement de mode de collaboration relèverait en effet de la pensée magique ou du discours incantatoire. Cependant, si le gestionnaire ne peut décréter la coopération, il peut toutefois s'employer à en favoriser l'émergence. La sociologie des organisations et la sociologie du changement offrent à cet égard des pistes intéressantes. À l'opposé des approches purement rationnelles, y sont proposés plusieurs modèles visant à susciter l'indispensable coopération des acteurs organisationnels en prenant explicitement en compte la problématique posée par l'interdépendance grandissante d'intérêts et de logiques différents, voire opposés. Que ce soit à travers des relations d'influence basées sur le pouvoir, à travers des principes ou valeurs acceptés de tous, ou encore à travers la traduction des différentes rationalités, la mise en œuvre de ce changement devra impliquer une restructuration des modes d'interaction et de régulation dans l'organisation ainsi que l'intégration des différences de rationalité et de logique qui y cohabitent ${ }^{6}$. Quelle que soit l'approche, il appartiendra donc au leader de créer un cadre de référence suffisamment partagé pour que chacun puisse y ancrer les modalités de sa coopération. La passion manifestée par le leader dans cet effort d'alignement pourra notamment contribuer à faire émerger au sein du collectif de travail une vision commune propice à l'intégration des multiples intérêts en présence.

\section{La passion au « travail »}

La passion peut s'avérer l'un des moyens pour parvenir à la coopération. Le terme " passion » est tra- 
ditionnellement défini comme un intérêt vif pour quelque chose. Il est régulièrement associé au domaine des loisirs. Néanmoins, le concept de «passion au travail» a fait son apparition dans le contexte organisationnel depuis quelques années et semble même avoir plus de vertu qu'il n'y paraît suggérant que la passion est une caractéristique fondamentale des leaders efficaces. La «passion au travail » est définie comme l'amour qu'un individu éprouve pour son travail ${ }^{7}$ Le leader "passionné » sera amoureux de son travail et y sera attaché, développant parallèlement un attachement et un engagement forts envers son organisation.

\section{De la passion au « travail » à l'alignement émotionnel}

Dans des environnements et des organisations en perpétuelles évolutions et mutations, le gestionnaire doit constamment viser et atteindre les objectifs individuels et collectifs qui lui sont fixés dans le cadre des stratégies corporatives. Afin d'atteindre ces objectifs, l'un des aspects essentiels à considérer est la capacité du gestionnaire à transmettre sa vision. Pour y parvenir, la passion s'avère être un instrument extrêmement puissant.

En effet, la passion permet au leader de créer l'alignement émotionnel nécessaire au changement. L'alignement émotionnel est défini par Haag comme «aligner les employés vers les mêmes buts et provoquer une interprétation partagée de ce qui se passe dans leur environnement $\gg{ }^{8}$. Une telle démarche nécessite impérativement une attention particulière de la part du leader pour tracer cette ligne directrice et donc amener les employés à collaborer à quelque niveau hiérarchique que ce soit au sein de l'organisation. La passion permet donc au leader de favoriser le partage d'une vision corporative, de développer, de créer et de mettre en place une action commune. Le leader "passionné " inspire et motive ses collaborateurs, tout niveau hiérarchique confondu. Il procure un sens de l'engagement et du travail, stimule la créativité, et n'a de cesse de trouver un consensus en alignant les intérêts des employés et ceux de l'organisation pour faire face notamment aux périodes de changement et de crise ${ }^{9}$. Mais avant tout, pour optimiser sa démarche, celuici se doit de «prêcher » par l'exemple et d'être naturellement perçu comme un leader sans forcer les individus à le suivre.

La passion permet au leader de créer

l'alignement émotionnel nécessaire au changement.

\section{La passion, une caractéristique à double tranchant}

La passion peut aussi révéler un côté plus obscur. Vallerant et $a l^{10}$ distinguent deux types de passions: la passion "harmonieuse » et la passion « obsessive ». Les « harmonieux» favorisent une adaptation saine tout comme la satisfaction des besoins intrinsèques liés à la tâche qui leur incombe en démontrant un fort engagement et une vision plus collective. En opposition, les « obsessifs » démontrent certaines difficultés à contrôler leurs pulsions, travaillant coûte que coûte. Malgré un engagement qui peut être élevé, la stimulation de l'équipe demeure plus difficile et l'alignement émotionnel parfois problématique.

Il en ressort deux types de "passion au travail» dichotomiques. Dans une logique d'alignement émotionnel et de vision partagée, le leader « harmonieux » apparait donc fondamentalement plus enclin à faciliter la création de cette ligne directrice collective qui aura pour effet de favoriser la coopération et par conséquent la résolution créative des problèmes.

$\underline{\text { Figure } 1 \text { : Modèle exploratoire de conduite du changement }}$

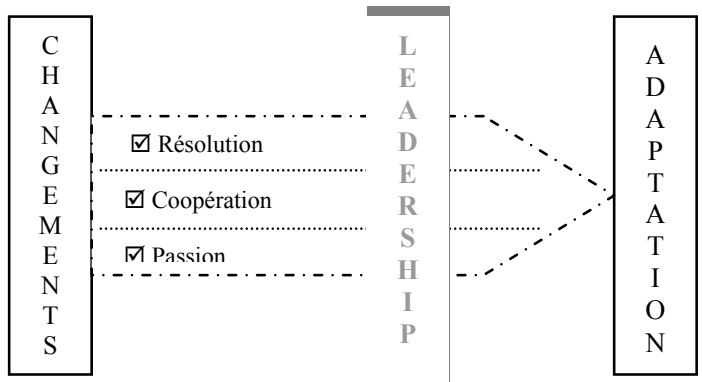




\section{Conclusion}

Le modèle exploratoire de conduite du changement présenté dans cet article et illustré ci-dessus (Figure 1) propose de considérer individuellement et conjointement la résolution créative des problèmes, la coopération et la passion comme trois variables de l'adaptation au changement, dans le but de mieux appréhender les nouveaux défis organisationnels. Tel qu'abordé précédemment, les organisations évoluent dans des environnements turbulents et instables qui les poussent à développer leur capacité d'adaptation afin d'assurer leur pérennité.

Afin d'optimiser cette démarche, le gestionnaire devrait idéalement être capable de s'appuyer sur ces trois variables interactives, faisant ainsi preuve de leadership dans sa conduite du changement, tout en favorisant un leadership «émergent ». Bien que le nombre de variables clés puisse être plus important, nous pensons que celles proposées sont fondamentales dans une telle logique d'adaptation à l'environnement en mutation. En somme, la passion contribue à favoriser la création de l'alignement émotionnel nécessaire à une coopération créative, et ce, dans la recherche de solutions adaptées aux nouveaux impératifs organisationnels, ce qui permet au passage d'optimiser cette démarche d'adaptation.

Le modèle exploratoire de conduite du changement proposé offre donc les bases théoriques pour de futures recherches empiriques qui permettraient de valider certaines hypothèses ou liens soulevés dans l'article tels que l'influence des trois variables clés (la résolution créative des problèmes, la coopération et la passion) et leur relation avec l'adaptation au changement. Ce champ d'études apparaît alors fort imposant et prometteur, laissant place à de nombreuses explorations possibles abordant le thème de la conduite du changement, élément central à toute réalité organisationnelle contemporaine.

\section{Notes et références}

1 Étymologiquement, le mot vecteur tire son origine du verbe latin vehere " transporter " et du nom vector " qui transporte ». Il est utilisé ici dans le sens de transmetteur.

2 Lichtenstein, B., Uhl-Bien, M., Marion, R., Seers, A., Orton, J.D.et Schreiber,C. (2006). « Complexity leadership theory: An interactive perspective on leading in complex adaptative systems ». E:CO, vol. 8, no 4. p. 212.

3 D'Zurilla, T.J., Nezu, A.M., et Maydeu-Olivares, A. (2004). «Social problem solving: Theory and assessment » dans Chang, E.C., D'Zurilla T.J. et Sanna, L.J. (Eds), Social problem solving: Theory, research, and training, p. 11-27, Washington, DC, American Psychological Association.

4 Hargadon, A.B. et Bechky, B.A. (2006). «When collections of creatives become creative collectives: A field study of problem solving at work, Organization Science, vol. 14, no 1, p. 484-500.

5 Zarafian O. (1995). La nouvelle productivité, Paris, L'harmattan, cité dans R. Soparno, (2005). « La coopération inter-individus : Une perspective par la théorie de l'apprentissage, le cas de la gestion de projet dans le secteur automobile ", Revue Internationale sur le Travail et la Société, vol. 3, no 2, p. 1080-1114.

6 Bernoux, P. (2004). Sociologie du changement dans les entreprises et les organisations. Paris, Éditions du Seuil.

7 Baum, J.R., Locke, E.A. et Smith, K.G (2001). «A multidimensional model of venture growth », Academy of Management Journal, vol. 44, p. 292-303.

8 Haag, C. (2007). « Le traitement des événements stratégiques dans le CODIR: Le rôle de l'intelligence émotionnelle du dirigeant », XVIe Conférence internationale de management Stratégique, Montréal, 6-9 juin.

9 Bernoux, P. (2004) op. cit.

10 Vallerand, R. J., Blanchard, C., Mageau, G. A., Koestner, R., Ratelle, C. F., Léonard, M., Gagné, M., \& Marsolais, J. (2003). « Les passions de l'Âme: On obsessive and harmonious passion. Journal of Personality and Social Psychology, vol. 85, p. 756-767. 


\section{Publicité}

«CRDT » 